\title{
REVIEW
}

\section{OF TRISKAIDEKAPHOBIA AND BOARD-FILLING GAMES WITH RANDOM-TURN ORDER}

\author{
Advances in Computer Games \\ $13^{\text {th }}$ International Conference, ACG 2011 \\ Tilburg, The Netherlands, November 2011 \\ Revised Selected Papers \\ Edited by H. Jaap van den Herik \& Aske Plaat \\ 2012, Springer-Verlag Berlin Heidelberg \\ ISBN 978-3-642-31865-8 \\ $54.00 €$
}

\section{Reviewed by Dap Hartmann}

These are the proceedings of the $13^{\text {th }}$ conference on Advances in Computer Games which was held in Tilburg in November 2011. The first eight conferences (1975-1996) dealt exclusively with Computer Chess, but after DEEP BLUE's victory over Kasparov in May 1997, the scope was broadened to include other games as well. The conference name was changed from Advanced in Computer Chess (ACC) to Advances in Computer Games (ACG) but the sequence numbering was kept. So, despite this being only the fifth truly ACG conference, it is referred to as ACG13.

While many tall buildings do not have a $13^{\text {th }}$ floor and most airplanes do not have a $13^{\text {th }}$ row, no such triskaidekaphobia plagued the organizers of this conference. This courageous attitude earns buyers of this book a nice dividend, because the ACG13 proceedings have 52\% more pages with $75 \%$ more illustrations than the ACG12 proceedings, and there are $45 \%$ more papers. And all that for a price that is only $12.5 \%$ higher. But more important than that, the quality of the contributions is at least as high as two years ago.

With a whopping total of 29 papers it is quite impossible to discuss all of them within the limited space of this review. Fortunately, the editors did a fantastic job of doing just that, and the result is the 9-page preface to this book. I strongly recommend that you read that preface first after buying this book, as it provides a good overview of the highlights and the great variety of all the papers in these proceedings. As usual, I have selected some of my personal highlights from these proceedings.

Maybe not a highlight, but rather a paper that puzzled me a bit is 'Infinite Connect-Four Is Solved: Draw' by Yoshiaki Yamaguchi, Kazumori Yamagichi, Tetsuro Tanaka, and Tomoyuki Kaneko. To show that ConnectFour played on an infinite board is a draw, the authors present a never-losing strategy that, when adopted by both players, invariably leads to a draw. I am not convinced that this warrants the conclusion that optimal play by both players always leads to a draw. Maybe there is some other strategy for player A which always leads to a win under optimal play of player B. This paper mainly proves that there is a strategy that always leads to a draw. But in any game a strategy can be outlined which, when consistently adopted by both players, always leads to a draw. As an extreme example, consider the game of Chess and the strategy that both players only move their knights between the first and the third rank. But maybe I am missing something? Correspondence is invited - a good test to see whether people actually read this review.

Ingo Althöfer is always exploring the fringes of computer game playing, and several of his original ideas have inspired other computer game researchers. For many years, Ingo has been actively promoting the Drei Hirn (three-brain) concept, where two computer programs assist one human expert in finding the best move in various games. He started this project way back in 1985, applying it to Chess at first. Playing many such games, Althöfer proved (estimated) that Drei Hirn consistently was some 200 Elo points stronger than the individual computer programs. Amongst the highlights of Drei Hirn Chess was the game against David Bronstein in the 1993 Aegon tournament (Bronstein won), and the Shuffle-Chess match (under tournament conditions) against Arthur Yusupov that Drei Hirn won by 5-3. Subsequently, Althöfer used the Drei Hirn concept in Backgammon, Othello, FreeCell, and Go. In 2004, he designed the game EinStein Würfelt Nicht! (EWN). The name of the game refers to the famous quote by Albert Einstein in a letter to Max Born in 1926 in which he expressed his doubts about the nature of quantum mechanics: "Quantum mechanics is certainly imposing. But an inner voice tells me that it is not yet the real thing. The theory says a lot, but does not really bring us any closer to the secret of the 'old one.' I, at any rate, am convinced that He does not throw dice." 
Many years later (1943) Einstein rephrased it to "As I have said so many times, God doesn't play dice with the world."

It must have been a wonderful feeling for Ingo to see Richard Lorentz from California State University present a paper on EinStein Würfelt Nicht! at this conference. In 'An MCTS Program to Play EinStein Würfelt Nicht!', Lorentz describes ONESTONE, a computer program that uses the Monte Carlo Tree Search (MCTS) approach to play EWN. ONESTONE plays a decent game against human opponents and is on equal par with EWN programs using mini-max based search. Despite this claim, Lorentz' EWN program WANDERER ended last (ex aequo with Althöfer's own program HANFRIED) in the $16^{\text {th }}$ Computer Olympiad which was held in conjunction with the $19^{\text {th }}$ World Computer Chess Championship (won by JUNIOR) and the ACG13 conference. The gold medal was for MeINSTEIN, written by Theo van der Storm (who sadly passed away in 2009) and Jan Krabbenbos. Nevertheless, I am convinced that, given his track record in Amazons (where he won the gold medal for the past four years) Richard Lorentz has a good chance to dominate the EWN tournament in the forthcoming Computer Olympiads.

Ingo Althöfer also presented a paper at this conference. In 'On Board-Filling Games with Random-Turn Order and Monte Carlo Perfectness', he describes a generalization of two-player zero-sum games where the flip of a fair coin determines which side moves next, and proves that there is an entire class of such games that are Monte Carlo perfect. In pure Monte Carlo, every legal move is evaluated by playing $T$ random games (until the end) starting with that move, and averaging the resulting scores. A game is called 'Monte Carlo perfect' when pure Monte Carlo converges to perfect play when $T$ goes to infinity. Althöfer shows that it applies to boardfilling games with random-turn order, but not to games like Amazons and Go. A legitimate question therefore is whether this result has any practical applications or whether it remains a purely academic matter. In what I would call typical Ingo Althöfer style, he turns this question around by encouraging his readers to "find interesting board-filling games with random turn order!" (his exclamation mark). I thought about that for a while, but I am afraid I lack the necessary creativity in that domain. It did get me wondering about one of the implications of random-turn order games, namely that one player could make two or more moves in a row. Statistically, on average once every 1000 turns, a player will make 10 moves in a row! Just to keep it simple, I thought about applying that concept to Chess, as that is the game that I am most familiar with. What kind of strategy would you have to use in situations where you (and your opponent!) could make several moves in a row? What about if you were allowed to make only two moves in a row? What if that was the rule - that you have to make two moves in a row at every turn? As with many original ideas, it turns out that this one was already taken. According to Wikipedia, a local newspaper in Marseilles published this idea in 1925. This Chess variant became known as Marseillais Chess, and in the 1930s it was quite popular with chess legends such as Alekhine and Réti.

This double-move chess got me thinking about the game of Arimaa, because in Arimaa a player is allowed to make one to four consecutive moves at every turn. The proceedings of ACG12 contained only one paper on Arimaa, and I ended my review of those proceedings with a request for more papers on the game of Arimaa. Unfortunately, no such contributions were submitted to this conference. Next time, anyone?

If I had more space, I would have liked to discuss some of the three 'serious games' that are included in these proceedings. If only for that curious label 'serious' which suggests that other games are not serious. Tell that to Kasparov or Lee Sedol (widely regarded as the current strongest Go player in the world), and see how they feel about that.

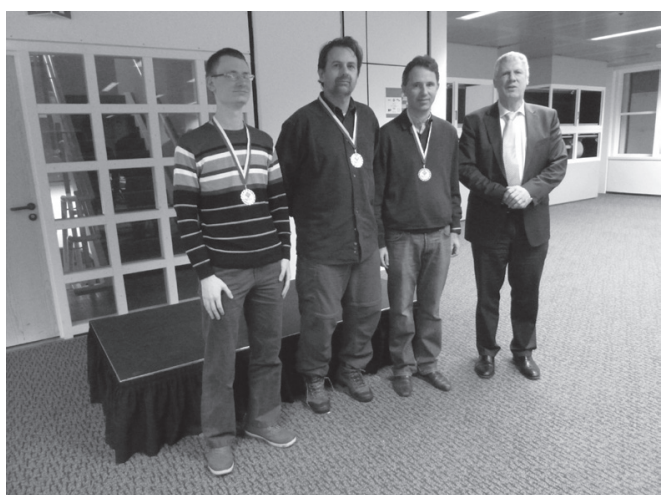

F.1.t.r.: Winners of the Hex Tournament (see pp. 124-127).

Broderick Arneson, Ryan Hayward, Tristan Cazenave, and Jaap van den Herik (TD). 